Punjab University Journal of Mathematics (2021),53(2),147-161

https://doi.org/10.52280/pujm.2021.530205

\title{
The Long-term structural effect of Coronal index solar activity on the ENSO and QBO Time series data using Fractal Dimension
}

\author{
Muhammad Fahim Akhter \\ Mathematical Sciences Research Centre, \\ Federal Urdu University of Arts, Sciences and Technology, Karachi, Pakistan, \\ Email: m fahimakhter@yahoo.com
}

Received: 20 April, 2019 / Accepted: 04 November, 2020 / Published online: 22 Feb-ruary, 2021

\begin{abstract}
The study concentrated on the fractal dimension of solar activity and climatic parameters. We analyzed comparatively for each parameter numerically. All values are estimated by Box Counting technique of fractal dimension. According to the theme of study, we used a monthly dataset of Coronal Mass Ejection (Coronal Index (CI)), ElNino Southern Oscillation (ENSO) and Quasi-Biennial Oscillation (QBO) from 1954 to 2016. The time seriesof ENSO and QBO are distributed according to the CORONAL INDEX (CI) cycles $(19,20,21,22,23$, and 24) to understand their relationship in the perspective of persistence or anti-persistence.The fractal dimension(D) represents the complexity and Hurst exponent $(\mathrm{H})$ indicates the long memory dependence of the selected time series, with scaling constant $(\mathrm{a}, \mathrm{c})$. The results obtained indicate the persistence $(1<D<1.5)$ for CORONAL INDEX (CI) with distributed ENSO and QBO cycles. The fractional Brownian motion ( $\mathrm{fBm}$ ) is also found long memory dependence $(1>H>0.5)$ and locally lowpass signal for all studied cycles observed.A linear relationship implies between Hurst coefficient and fractal dimension for a statistical assumption $(H+D=2)$. The fractal scaling instrument is established between the global indices (ENSO \& QBO) and solar activity (particularly CORONAL INDEX (CI)) cycles, the inverse correlation with ENSO and direct with QBO are observed. The results obtained in this research work may help to describe the solar-terrestrial relationship.
\end{abstract}

Key Words: Coronal Mass Ejection, El-Niño Southern Oscillation, Quasi-Biennial Oscillation, Fractal Dimension, Hurst Exponent, Fractional Brownian Signal.

\section{INTRODUCTION}

Sun has an important role in driving the space weather. When the sun magnetic vibration occurs on behalf of occurrence various energy ejects out in space and reached out towards the earth. The two main solar activities Coronal mass ejection and solar flare are 
also responsible for bringing the variation in space and earth weather $[12,19]$. Coronal mass ejection from the solar corona is an important part of solar activity. There are two types of CMEs Hallo's one is partial Hallo and another is full Hallo. The full Hallo is a complete disk outside the sun and follows the sunspot cycle than partial Hallo. Recently a theory was presented as coronal index activity observed as a good candidate to express solar activity especially with sunspot [2]. Various studies are established with a strong relationship between solar activities and global indices. In this communication coronal index solar activity compares with global parameters using fractal dimension. Coronal mass ejection occurs in the close region of the magnetic field that overlies the inversion line of the magnetic field [16]. The total energy at wavelength $530.3 \mathrm{~nm}$ within Fe XIV spectral emission line is emitted by sun corona called the outermost atmospheric layer of the sun [1]. It is acknowledged that sun energy contributes to the earth's climate system (Rind 2002). The interaction of sun-earth is considered solar-terrestrial astrophysics, through the emission of particles, magnetic fields and electromagnetic radiation from one place to another [13, 15 and 21]. Astrophysical processes are highly nonlinear over wide ranges of spatial and temporal scales [20 and 31]. In performing solar activity almost nothing happens in solar minimum while the explosion of solar maximum causes to disturb the space weather and engineering system on earth and space. These peaks are opposite extremes (maximum and minimum) of solar activity. The maximum and minimum solar activity rotates with global analogue El Niño and La Niña. The extreme terrestrial analogue El Niño and La Niña is an opposite extreme of Pacific oscillation. In the period of every 2-7 years, the condition of surface waters across the Pacific Equatorial Ocean is warm and cool. The warm episode is El Niño and the cool episode is La Niña. In a piece of information, each one (El Niño and La Niña) has its regional effect which is varied, like the extremes of the sun [11]. Similarly, in the detection of QBO jointly with several solar indices (sunspot number, CaII area and $\mathrm{K}$ index, $10.7 \mathrm{~cm}$ flux, coronal green line) are discussed and found the $1.3 \mathrm{yrs}$ periodicity. periodicity in the correlation of the green line intensity and magnetic field in the lower corona, also the QBO revealed with green line coronal emission with time in the range of 1.5-4yrs [4]. The cycles range on timescales shorter than 1 yr.to the mid-term cycles or so-called quasi-biennial oscillations (QBO) with a typical length of 1-4yr are studied in the solar dataset by [25 and 34]. The influence of solar activity on the temperature in the stratosphere ( $10-50 \mathrm{~km}$ ) is hard to see in the QBO phase. The QBO is approximately 2 years of oscillations wind in the equatorial lower stratosphere [3]. The oscillation of zonal wind and temperature is mainly found in the tropical stratosphere, the irregular oscillation slightly longer than two years is called (QBO) [4, 31 and 20]. The reversal wind in the stratosphere over the equator is alternate of westerly and easterly wind oscillation around 28 months. The statistical relationship between solar flare and the troposphere circulation mechanism was represented in [31]. The mechanism finds which energetically weak signal of solar variability can influence the condition in the lower atmosphere. According to solar flare theory, the particles interact with some greenhouse gases in the upper troposphere and lower stratosphere, first to change radiation balance than to anomalies in the pressure patterns and then the atmospheric circulation. The other more convincing evidence of solar weather is presented by Labitzke and Van Loon [20]. They claimed QBO may be a possible factor that modulates by solar effects on the atmosphere. Their work is based on the $10.7-\mathrm{cm}$ solar record of the winter temperature at $30 \mathrm{mb}$ during the last three solar cycles. 
The sunspot correlation is very low according to QBO westerly and easterly phase but in winters temperature at $30 \mathrm{mb}$ are found strongly correlated. In the United States, a record of solar activity pointed clearly to a severe winter through December 1989 from 1998 at the west phase of QBO. In winter overall was mild except February. Although the solar-QBOweather relationship is new important, and unexplained in climate diversity. ENSO theories are utilized to understand the diversity of climate and sun. There are several theories (statistical and stochastic time series analysis) that are suggested to define this diversity.Solar forcing, modulated by the $11 \mathrm{yr}$ solar activity and its intensity was illustrated as a nonlinear system of seasonal forcing, like a dominant factor causing in-contrast of regional SSTs timescales studied by [38].Similarly, in one more study, these caused by a solar forcing at a frequency of 1.0 cycle/yr. and these periodic features are observed against global climate data and found significant understanding for El Niño/La Niña phenomenon[6].The mechanism of the existence of solar activity and climate also argue in a paper to fully understood nonlinear system (UV radiation, solar wind, and galactic cosmic rays being the most promising candidates)[23].The periodicities analysis with the help of wavelet observed as a fundamental solar cycle is interpreted as simply harmonics from underlying sunspot magnetism phenomena [37]. We suggest fractal dimension schemes in studied parameters and wavelet base fractional Brownian motion utilized to understand high and low frequencies. The iterative schemes of fractal dimension attractors are defined in [20 and 31]. The significant relationship between sunspots and corresponding ENSO time series data is established [2]. These two events (ENSO and QBO) play a role in local weather and overall climate. The variation of these events causes for global warming, hurricane, droughts, and flood, etc. Due to the occurrence of solar activity may be an increase or decrease in the effects of these events on earth climate. It is necessary to understand the structural dynamics of solar and climatic data for future prognosis. In this paper, we study long-range dependence (LRD) and with the help of the Hurst exponent check Brownian signal of the parameters. The used value of the Hurst coefficient $(\mathrm{H})$ and fractal dimension (D) is used to estimate indexing parameters (CORONAL INDEX (CI), ENSO, and QBO) for length profile. While the scope of this paper is to identify long memory dynamics relationship.

\section{Material And Methods}

The research work in this manuscript utilizes the CORONAL INDEX (CI) from 1954 to 2016 along with the ENSO and QBO time series data. The distributed cycles are (1954.081964.09), (1964.09 - 1976.04), (1976.04 - 1986.06), (1986.06 - 1996.03), (1996.03 $2008.09)$, and $(2008.09-2016)$ respectively. The research work done in this manuscript includes the scheme of nonlinear scale $\left(a \times x^{D}+c\right)$ is furnished in (sec: 2.1.1) and utilizes the Box dimension or box-counting technique to understand the quasi-regular (persistent) behavior of each selected duration. While we set estimate De, a roughness parameter, and $\mathrm{He}$, a long memory parameter dynamics estimated using fractals software.

2.1. Fractal Dimension of the CORONAL INDEX (CI)s, ENSO and QBO Activities. TheClimatic indices such as ENSO,IOD, SST, QBO etc.,varyover many time scales.The climatic indices time series along with the CORONAL INDEX (CI) time series can be representedby a time fractal curve, so fractal methods are used to establish the order of quasi regularity and irregularity and hence be possible to understand the self-similarity at 
various time scales. There are two approaches elaborated for fractal analysis, first one elaborated by [21] for determining time series, geometric curve and the length of time scaling curve. According to [21] and [14],H relates to the value of fractal.

$$
H+D=2
$$

The second approach was elaborated by $[3,8,21,35]$, the representation of the time series of general Brownian function and determining the process of time scaling and its statistical characteristics by fractal methods. These functions, statistical characterize the process of time-scaling by self-affinity [18, 29].The technique used for fractal dimension is a nonlinear box-counting technique, which is a traditional and a more useful technique. The complex system is using fractal geometry science understand nature is a similar-affine or self-similar feature. The application of scaling properties of Astrophysical time series data as a driver for the development of new concepts, notions, formalisms and techniques is advantageous [4 and 30].

2.1.1. Fractal Dimension in using Box Counting Method. The grid base technique followed in the box-counting method. The image or time-series data can be selected by the grid and count how many grid boxes are covering the part of the image or time-series data. By repeatedly shrinking the size of the grid, the structure pattern may be captured more accurately this technique of fractal dimension shown in equation (2.2). Where the number of boxes represents by $\mathrm{N}$ and covering the pattern $\mathrm{r}$ is the magnification or the inverse of the boxes, on the graph the slope of $\mathrm{Y}$-axis $\log (\mathrm{N})$ against $\log (\mathrm{r})$ on the $\mathrm{X}$-axis this ratio label by fractal dimension (D) [7, 14, 28].

$$
D=\frac{\log (N)}{\log (r)}
$$

In fractals software number of equations and techniques are available to approximate the empirical curve, we used equation (2.3), the measurement equation of fractal law $N(x)=$ $X^{D}$, which permit to measure also the fractal law deviation by a nonlinear equation[28].

$$
N(x)=a X^{D}+c
$$

Where 'D'represents the fractal dimension, 'c' corresponds to the point of origin of the Y-axis and 'a' is called the "pre-factor of shape".

2.1.2. Hurst Exponent Associated to Fractal Dimension Scale. The Hurst exponent is used in various applied mathematical areas, with the help of fractals and chaos theory, long memory processes (LRD) and spectral analysis are used to calculate the long term dependencies of time series data [17]. It is a global phenomenon to estimate the smoothness of time series data, which is related to the fractal dimension [26]. Hurst exponent is a concern for important world phenomena, for example, climatic dynamics [24], astronomy and astrophysics [19], economy [10], geomagnetic field [33, 34], plasma turbulence [9] and traffic traces [5]. The importance measured of neuron networks in the body, the emitted color of the sun, the surface of the sea during a storm, dust in the air, clouds, distribution of frequencies, trees, etc., are attached with fractal dimension [14]. The scale coefficient H-value is 0.5 (series purely random uncorrelated) If $1>H>0.5$ (series random walk, persistent and positive auto correlation (fBm). If $H<0.5$ (series cover less distance random walk, anti-persistent and negative correlated (fBm)) while the Hurst exponent is 1 for periodic motion [24 and 
36].The Box-counting or box dimension technique is a popular technique to calculate mathematical and empirical estimation with ease [7].The main concerns of these analyses are obtained on more insight into the complexity and structure of the system as defined in [22]. Under certain limiting assumptions summarize the relationship represents in [32] is given below.

\begin{tabular}{cccc}
\hline \hline $\mathrm{H}$ & $\mathrm{D}$ & Correlation & Nature of process \\
\hline$>0.5$ & $<1.5$ & Positive & Persistent \\
$=0.5$ & $=1.5$ & Zero & Brownian \\
$<0.5$ & $>1.5$ & Negative & Anti-Persistent height
\end{tabular}

' $\mathrm{H}$ ' is directly related to $\mathrm{D}$, in the modern approach, $\mathrm{H}$ is estimated by $\mathrm{D}$. The modern approach for calculating the Hurst exponent comes from fractal mathematics. With the help of ( $\mathrm{H}$ and $\mathrm{D})$, long-range dependence (LRD) and roughness or complexity of monthly time series (CIs, ENSO, and QBO) are observed and discussed in the next section.

\section{RESULTS AND DISCUSSION}

In this paper,the long-range dependence (LRD) relation is observed using $\mathrm{H}$ (Hurst exponent) with the help of relation $(H=2-D)$. Where $\mathrm{D}$ (fractal dimension) confine roughness (smoothness) and complexity of the studied system. The studied parameters (CI, ENSO, and $\mathrm{QBO})$ checked by $(\mathrm{H}$ and $\mathrm{D})$ values and based on $\mathrm{H}$ values fractional Brownian signal $(\mathrm{fBm})$ generated a sample path of the signal, 1000 samples. According to $\mathrm{fBm} H>0.5$ represents long-range dependence and if $\mathrm{H}_{j} 0.5$ represents intermediate dependence. $\mathrm{fBm}$ is a continuous-time Gaussian process which is followed $0<H<1$. In this study, different peaks and length of CI cycle (minimum 10.1 years and maximum 12.6 years) observed and corresponding (ENSO and QBO) monthly data in the same respect of CI cycles is used. During all study parameter periods, the $\mathrm{H}$ values $(0.52 \mathrm{H} 0.98)$ is observed. That is based on (LRD) parameter indicates themost of the series persistent and this tendency will remain for long future time-period. Consequently, D values $(1<D<1.5)$ is obtained which is a positive correlation of the process mean persistent (quasi-regular). Separate H and D values of CI, ENSO, and QBO furnished in Table 1-3.

The solar activity parameter is done in Table 1 . According to CI cycles, Hurst exponent values displayed between $0.5<H_{C . I}<1$. The manifested results understand the long future period of the CI cycles i.e., high present values followed high future value (most of the part persistent). In the duration of the $\mathrm{CI}$ cycle, 19 has high concentrated $\mathrm{H}$ value 0.928 , while among six CI cycles, cycles 23 concentrate low $\mathrm{H}$ value 0.685 . Similarly, CI cycle complexity (roughness) observed between $1.072 \leq D_{C . I} \leq 1.315$, that is positive autocorrelation of the dynamical process. The $\mathrm{CI}$ cycles are continuous-time Gaussian process that is why fBm follows $0<H<1$, along with CI cycles shows low pass $\mathrm{fBm}$ signal of each CI cycles exhibit long-range dependence depicted in Fig 1.In connection with sun-Earth, the climatic parameter ENSO and QBO are discussed in contrast to solar activity (CI) parameter. ENSO 3.4 is the most effective climatic region that is overlapping in the region ( 3 and 4). It is important to understand the structural behavior of this time series data because it is 
a consequence of drought and flood.The (H and $\mathrm{D})$ values are used to understand the complexity and LRD of ENSO data. Using non-linear scale persistent behavior of each ENSO cycles and total data is observed depicted in Table 2. The ENSO total dataset HURST and FRACTAL values $\left(H_{E N S O 3.4}=0.571\right.$ and, $\left.D_{E N S O 3.4}=1.48\right)$ are observed that is a positive correlation of ENSO total dataset. In the case of each cyclic behavior, ENSO preserved persistency i.e., $0.52 \leq H_{E N S O 3.4} \leq 0.898$ and $1.102 \leq D_{E N S O 3.4} \leq 1.48$. It is clearly understood that ENSO contains positive correlation and long-range dependence (LRD). ENSO fBm signal confines on low signal processing that is why the Hurst exponent range lies between $0<H_{E N S O 3.4}<1$, the low processing signal define long-term dynamics of the study parameter and the fBm is self-similar in distribution is depicted in Fig 2. The other climatic parameter QBO also studied in contrast to CI using Hurst exponent and fractal dimension. The monthly $30 \mathrm{mb}$ wind data used to understand the dynamics of QBO data along with distributed cycles. The important effect of QBO relates to hurricanes in the Atlantic and widely used for hurricane forecast, and also effective in cyclone and monsoon rainfall. Using HURST and FRACTAL dimension persistency observed in cycles and total data range depicts in Table 3. In total QBO data, the HURST and FRACTAL values $(H>0.5$ and $D<1.4)$ observed showing a positive correlation, less roughness and LRD process. In-case of cycles the Hurst and FRACTAL lower and higher concentrated values lie $(0.63 \leq H \leq 0.97$ and $1.023 \leq D \leq 1.37)$ is observed. That thing established the split cycles of QBO corresponding to CI shows long-range dependence (LRD) and indicates quasi-regular behavior that prolongs the forecast. The QBO signal processing (fBm) demonstrates the continuous-time Gaussian series that is why the Hurst values lie between $(0.5<H<1)$. Similarly, strong low pass QBO signal defines long-range dependence and self-similarity of the process depicts in Fig 3 .

The comparative study of cycles and total dataset using non-linear scales of fractal regimes showing highly persistency in coronal index behavior, in ENSO and QBO index furnished in Table 4. The $\mathrm{H}$ value is 0.77 in the total length of CI while ENSO and QBO greater than 0.5 observed that exhibits random walk and cover more distance. While the fractal value 1.23 in the total length of CI less rough (complex) than ENSO and QBO are greater 1.4 and lesser 1.5. It indicates the total data series have regularity, among all parameters $\mathrm{CI}$ is smoother than ENSO and QBO, ENSO and QBO are similar smoothness in total data series cycle (19-24).In utilizing a particular scale $\left(a \times X^{D}+c\right)$ persistency observed in all study structure (CIs, ENSO and QBO).Regarding (H and D) all the dynamics of study parameters are positively correlated in their behavior. Moreover,we develop the correlation instrument between the fractal structure of CIs, ENSO and QBO. Using fractal correlation instrument between parameters cycles, including total range. The long-term relation dependency of ENSO on CIs solar activity with a correlation (-0.02736). It indicates higher solar activityweaker and less frequent El Niño activity. The minimum cycle phase may more frequent in the generation of El Niño activity. Similarly, in long term relation of QBO on CIs solar activity with a correlation $(0.81398)$ showing QBO modulation around the maximum year by sun intensity was more energetically enhanced. The long-range dependence relation of CI with ENSO and QBO is furnished in fig 4. Cycles fractals showing the CI and QBO is gradually moving together than ENSO that shows CI and QBO dynamics are positively correlated while with ENSO cycles shows negative dynamics with CI. 


\section{CONCLUSiON}

The study comprises the Box counting technique for nonlinear scaling regime analysis. Although Box counting or Box dimension is a traditional method for obtaining the fractal dimension and Hurst exponent,it gives better information about the dynamics of the time series as compared to the others. These findings indicate persistency in the range $0.5<H<1$. The results obtained are a very high degree of persistent behavior Coronal Index (CI) and the Hurst exponent of durations are declining trend, a slightly less persistent behavior in ENSO, but got a positive slope in cyclic behavior and comparatively less similar persistent behavior in QBO, it negatively oscillates trend behavior depicts in fig 4 . Several exponents of cycles are above 0.8 and some are below 0.8 , namely the corresponding time-series parts were very persistent demonstrated in fig 4. Using fractal dimension the instrument developed between Coronal Index (CI) and ENSO cycles, including total data shows a negative correlation (-0.2736). The instrument between Coronal Index (CI) and QBO cycles represents positive correlation (0.82899). Based on the above discussions, we conclude that the Coronal Index (CI) activity cycle data harms the ENSO time series data.It is established that if solar activity maximum than climatic parameters (ENSO) activity was found weaker under the long-range dependence relation. While QBO modulation was more important during periodicities around the maximum intensity of the solar activity, where the solar cycle enhanced during activity maximum under long-range fractal relation. Other solar activity indices may have a similar impact on the other climatically index data. In almost all cases it was found that the dynamics are long-memorized,the order of regularity and also self-similarity.

\section{ACKNOWLEDGMENTS}

The research work done in this paper is a part of the doctoral thesis of the author which was completed under the supervision of Dr. Shaheen Abbas. The author is thankful to the NOAA for providing solar Activity and global indices data with the following data sources.

https://www.ngdc.noaa.gov/stp/solar/corona.html

http://www.suh.sk/online-data/modifikovany-koronalny-index

http://www.cpc.noaa.gov/products/analysis_monitoring/ensostuff/ONI ${ }_{c}$ hange.shtm1

http ://www.esrl.noaa.gov/psd/data/climateindices/list/

\section{REFERENCES}

[1] M. F. Akhter, S.Abbas, D. Hassan, Study of Coronal Index Time Series Solar Activity Data in the Perspective of Probability Distribution. Proceeding of Pakistan Academy of Sciences: A Physical and Computational Sciences 55(1) (2018) 27-34.

[2] M. F. Akhter, S. Abbas, D. Hassan, The relationship of periodic behaviour between coronal index cycles and associated ENSO by using Markov process. Astrophysics and Space Science, 364(7) (2019) 107.

[3] M. P. Baldwin, L. J. Gray, T. J. Dunkerton, K. Hamilton, P. H. Haynes, W. J. Randel, ... D. B. A. Jones, The quasi-biennial oscillation. Reviews of Geophysics, 39(2) (2001) 179-229.

[4] G. Bazilevskaya, A. M. Broomhall, Y. Elsworth, V. M. Nakariakov, Space Sci. Rev., 186, 359 (2014).

[5] ] G. Dattatreya, Hurst parameter estimation from noisy observations of data traffic traces. In 4th WSEAS International Conference on Electronics, Control and Signal Processing, Miami, Florida, USA , (2005) 1719. 
[6] D. H. Douglass, R. S. Knox, The Sun is the climate pacemaker I. Equatorial Pacific Ocean temperatures. Physics Letters A, 379 (2015) 823-829.

[7] K. Falconer, Fractal geometry: mathematical foundations and applications. John Wiley Sons (2004).

[8] M. Feder, E. Weinstein, Parameter estimation of superimposed signals using the EM algorithm. IEEE Transactions on Acoustics, speech, and signal processing, 36(4) (1988) 477-489.

[9] M.Gilmore, C. X. Yu, T. L. Rhodes, W. A. Peebles, Investigation of rescaled range analysis, the Hurst exponent, and long-time correlations in plasma turbulence. Physics of Plasmas (1994-present), 9(4) (2002) 1312-1317.

[10] M. S. Granero, J. T. Segovia, J. G. P'erez, Some comments on Hurst exponent and the long memory processes on capital markets. Physica A: Statistical Mechanics and its Applications, 387(22) (2008) 5543-5551.

[11] M. Guhathakurta, T. Phillips, The Solar Cycle Turned Sideways.Space Weather, 11(5) (2013) 212-213.

[12] N. Gyenge, A. Ludm 'any, T. Baranyi, Active longitude and solar flare occurrences. The Astrophysical Journal 818.2 (2016) 127.

[13] J. K .Hargreaves, The solar-terrestrial environment, Cambridge (1992).

[14] D. Hassan, S. Abbas, M. R. K. Ansari, B. Jan, Solar Flares Data Analysis on the application of Probability Distributions and Fractal Dimensions and a comparative analysis of North-South Hemispheric Solar Flares Data Behavior (2014).

[15] H. M. Hasting, G. Sugihara, Fractals A Users' Guide For The Natural Sciences, Oxford University Press (1993).

[16] A. J. Hundhausen, Sizes and locations of coronal mass ejections: SMM observations from 1980 and $1984-$ 1989. Journal of Geophysical Research: Space Physics (1978-2012), 98(A8) (1993) 13177-13200.

[17] H. E. Hurst, R. P. Black, Y. M. Simaika, Long-term storage: an experimental study, Constable (1965).

[18] P. G. Kapiris, K. A. Eftaxias, K. D. Nomikos, J. Polygiannakis, E. Dologlou, G. T. Balasis, ... V. E. Hadjicontis, Evolving towards a critical point: A possible electromagnetic way in which the critical regime is reached as the rupture approaches. Nonlinear Processes in Geophysics, 10(6) (2003) 511-524.

[19] A. Kilcik, C. N. K. Anderson, J. P. Rozelot, H. Ye, G. Sugihara, A. Ozguc, Nonlinear prediction of solar cycle 24. The Astrophysical Journal, 693(2) (2009) 1173.

[20] K. Labitzke and H. van Loon, Association between 11-year solar cycle, the QBO, and the atmosphere. Part I: The troposphere and the stratosphere in the northern hemisphere in the winter. J. Atmos. Terr. Phys. 50 (1988) 197-206.

[21] B. B. Mandelbrot, The Fractal Geometry of Nature, Freeman, New York (1983).

[22] ] F. Michael Barnsley,: Fractals everywhere. Academic Press, ISBN 978-0-12-079062-3, pp. I-XII, 1394(1998)

[23] Le. Mou J.el J.-L., F. Lopes, V. Courtillot, A solar signature in many climate indices. Journal of Geophysical Research, 124 (2019) 2600-2619.

[24] D. Nikolopoulos, E. Petraki, N. Temenos, S. Kottou, P. H. Yannakopoulos, Hurst exponent analysis of indoor radon profiles of Greek apartment dwellings (2014).

[25] K. Ol'ah, Zs. Kovari, K. Petrovay, W. Soon, S. Baliunas, Z. Kollath, K. Vida, Magnetic cycles at different ages of stars. Astronomy Astrophysics 590 (2016) A133.

[26] T. L'opez, C. L. Mart'inez-Gonz'alez, J. Manjarrez, N. Plascencia, A. S. Balankin, Fractal analysis of EEG signals in the brain of epileptic rats, with and without biocompatible implanted neuroreservoirs. In Applied Mechanics and Materials, 15 (2009) 127-136.

[27] S. Rehman, A. H. Siddiqi, Wavelet based Hurst exponent and fractal dimensional analysis of Saudi climatic dynamics. Chaos, Solitons Fractals, 40(3) (2009) 1081-1090.

[28] N. Scafetta, P. Grigolini, Phys. Rev. E, 66 (2002) 036130.

[29] I. I. Salakhutdinova, A fractal structure of the time series of global indices of solar activity. Solar Physics, 181(1) (1998) 221-235.

[30] J. D.Scargle, Astronomical Time Series Analysis, in Errors, bias and uncertainties in astronomy, Jaschek C Murtagh F(eds), Cambridge University Press (1990).

[31] CJE. Schuurmans, The influence of solar flares on the tropospheric circulation, KNMI Mededelingen en Verhandelingen 92, 123, De Bilt, The Netherlands (1969).

[32] G. Sugihara, R. M. May, Nonlinear forecasting as a way of distinguishing chaos from measurement error in time series. Nature, 344(6268) (1990) 734. 
[33] N. A. Smirnova, M. Hayakawa, Fractal characteristics of the ground-observed ULF emissions in relation to geomagnetic and seismic activities. Journal of Atmospheric and Solar-Terrestrial Physics, 69(15) (2007) 1833-1841.

[34] W. Soon, V. M. Velasco Herrera, R. G. Cionco, S. Qiu, S. Baliunas, R. Egeland, G. W. Henry, I. harv 'atov 'a, Monthly Notices of the Royal Astronomical Society, 483 (2019) 2748-2757.

[35] H. E. Schroeder, Biological problems of regenerative cementogenesis: synthesis and attachment of collagenous matrices on growing and established root surfaces. In International review of cytology, Academic Press, 142 (1992) 1-59.

[36] V. Suyal, A. Prasad, H. P. Singh, Nonlinear time series analysis of sunspot data. Solar Physics, 260(2) (2009) 441-449.

[37] V. M. Velasco Herrera, J. P'erez-Peraza, W. Soon, J. C. M'arquez-Adame, New Astron. , 607 (2018).

[38] $\mathrm{H}$. Weng, The influence of the 11 yr solar cycle on the interannual-centennial climate variability. Journal of Atmospheric and Solar-Terrestrial Physics 67 (2005) 793-805. 


\section{Tables and Diagrams}

TABLE 1: Fractal Dimension $\left(\mathrm{D}_{\mathrm{e}}\right)$ estimated of Coronal Index $(\mathrm{CI})$ by $\left(a \times X^{D}+c\right)$ and calculated $(H e)$ by $H_{e}=2-D_{e}$

\begin{tabular}{|c|c|c|c|c|c|c|}
\hline Cycle & Duration & $\mathbf{H}_{\mathrm{CI}}$ & $\mathbf{D}_{\mathrm{CI}}$ & Equation & Mean & S.D \\
\hline $\mathbf{1 9}$ & $1954.08-1964.09$ & 0.928 & 1.072 & $1.1002 x^{1.072}-0.034$ & 9.394 & 6.272 \\
\hline $\mathbf{2 0}$ & $1964.09-1976.02$ & 0.766 & 1.234 & $1.027 x^{1.234}+0.017$ & 6.9946 & 3.849 \\
\hline $\mathbf{2 1}$ & $1976.04-1986.06$ & 0.821 & 1.179 & $1.009 x^{1.179}+0.017$ & 8.685 & 5.0881 \\
\hline $\mathbf{2 2}$ & $1986.06-1996.03$ & 0.794 & 1.206 & $1.020 x^{1.206}+0.015$ & 9.004 & 5.489 \\
\hline $\mathbf{2 3}$ & $1996.03-2008.09$ & 0.685 & 1.315 & $0.987 x^{1.315}+0.019$ & 5.902 & 3.19 \\
\hline $\mathbf{2 4}$ & $2008.09-2016.12$ & 0.723 & 1.277 & $1.001 x^{1.277}+0.057$ & 1.749 & 0.876 \\
\hline $\mathbf{1 9 - 2 4}$ & $1954.08-2016.12$ & 0.77 & 1.23 & $1.041 x^{1.23}+0.002$ & 7.7918 & 4.8193 \\
\hline
\end{tabular}

TABLE 2: Fractal Dimension $\left(D_{e}\right)$ of ENSO estimated by $\left(a \times x^{D}+c\right)$ and calculated $\left(H_{e}\right)$ by $H_{e}=2-D_{e}$

\begin{tabular}{|c|c|c|c|c|c|c|}
\hline Cycle & Duration & $\mathbf{H}_{\text {C.I }}$ & $\mathbf{D}_{\text {C.I }}$ & Equation & Mean & S.D \\
\hline $\mathbf{1 9}$ & $1954.08-1964.09$ & 0.8823 & 1.1177 & $0.954 x^{1.117}-0.064$ & -0.387 & 0.678 \\
\hline $\mathbf{2 0}$ & $1964.09-1976.02$ & 0.52 & 1.48 & $0.920 x^{1.48}+0.048$ & -0.456 & 0.894 \\
\hline 21 & $1976.04-1986.06$ & 0.586 & 1.414 & $0.876 x^{1.414}+0.072$ & -0.064 & 0.651 \\
\hline $\mathbf{2 2}$ & $1986.06-1996.03$ & 0.6231 & 1.3769 & $0.921 x^{1.376}-0.088$ & 0.160 & 0.798 \\
\hline 23 & $1996.03-2008.09$ & 0.898 & 1.102 & $1.065 x^{1.102}-0.076$ & -0.069 & 0.852 \\
\hline 24 & $2008.09-2016.12$ & 0.8588 & 1.1412 & $0.948 x^{1,141}-0.068$ & 0.042 & 0.870 \\
\hline \multirow{2}{*}{$\mathbf{1 9 - 2 4}$} & $1954.08-2016.12$ & 0.517 & 1.483 & $0.944 x^{1.483}+0.011$ & -0.138 & 0.827 \\
\hline
\end{tabular}


TABLE 3: Fractal Dimension $\left(D_{e}\right)$ of QB0 estimated by $\left|a \times x^{D}+c\right|$

and calculated $H_{e}$ by $H_{e}=2-D_{e}$

\begin{tabular}{|c|c|c|c|c|c|c|}
\hline Cycle & Duration & $\mathrm{H}_{\text {Сво }}$ & $\mathbf{D}_{\text {Qво }}$ & Equation & Mean & S.D \\
\hline $\mathbf{1 9}$ & $1954.08-1964.09$ & 0.977 & 1.023 & $1.0003 x^{1.023}-0.019$ & -3.499 & 9.764 \\
\hline $\mathbf{2 0}$ & $1964.09-1976.02$ & 0.641 & 1.359 & $1.060 x^{1.359}+0.008$ & -3.498 & 11.422 \\
\hline $\mathbf{2 1}$ & $1976.04-1986.06$ & 0.866 & 1.134 & $1.071 x^{1.134}-0.031$ & -2.367 & 11.640 \\
\hline $\mathbf{2 2}$ & $1986.06-1996.03$ & 0.691 & 1.309 & $1.086 x^{1.309}-0.018$ & -2.775 & 10.877 \\
\hline $\mathbf{2 3}$ & $1996.03-2008.09$ & 0.63 & 1.37 & $1.048 x^{1.37}-0.0142$ & -4.305 & 13.717 \\
\hline $\mathbf{2 4}$ & $2008.09-2016.12$ & 0.691 & 1.309 & $1.049 x^{1.309}-0.0132$ & -2.763 & 14.684 \\
\hline $\mathbf{1 9 - 2 4}$ & $1954.08-2016.12$ & 0.546 & 1.454 & $1.078 x^{1.454}+0.0014$ & -3.300 & 12.096 \\
\hline
\end{tabular}

TABLE 4: Comparisons of Coronal Index (CI), ENSO and QBO.

\begin{tabular}{|c|c|c|c|c|c|c|c|}
\hline \multicolumn{2}{|c|}{ Parameters } & \multicolumn{2}{c|}{$\begin{array}{c}\text { Coronal Index } \\
\text { (CI) }\end{array}$} & \multicolumn{2}{c|}{ ENSO } & \multicolumn{2}{c|}{ QBO } \\
\hline Cycle & Duration & $\mathbf{H}_{\text {C.I }}$ & $\mathbf{D}_{\text {C.I }}$ & H $_{3.4}$ & $\mathbf{D}_{3.4}$ & $\mathbf{H}_{\text {QB }}$ & D $_{\text {QBO }}$ \\
\hline $\mathbf{1 9}$ & $1954.08-1964.09$ & 0.928 & 1.072 & 0.8823 & 1.1177 & 0.977 & 1.023 \\
\hline $\mathbf{2 0}$ & $1964.09-1976.04$ & 0.766 & 1.234 & 0.52 & 1.48 & 0.641 & 1.359 \\
\hline $\mathbf{2 1}$ & $1976.04-1986.06$ & 0.821 & 1.179 & 0.586 & 1.414 & 0.866 & 1.134 \\
\hline $\mathbf{2 2}$ & $1986.06-1996.03$ & 0.794 & 1.206 & 0.6231 & 1.3769 & 0.691 & 1.309 \\
\hline $\mathbf{2 3}$ & $1996.03-2008.09$ & 0.685 & 1.315 & 0.898 & 1.102 & 0.63 & 1.37 \\
\hline $\mathbf{2 4}$ & $1954.08-2016.12$ & 0.723 & 1.277 & 0.8588 & 1.1412 & 0.691 & 1.309 \\
\hline $\mathbf{1 9 - 2 4}$ & $1954.08-2016.12$ & 0.77 & 1.23 & 0.517 & 1.483 & 0.546 & 1.454 \\
\hline
\end{tabular}


Fig.1 A Strong low-frequency signal is obtained with the help of Hurst value( $\mathrm{H})$, in using $(\mathrm{fBm})$, that shows the long term dynamics of $\mathrm{CI}$ (cyclic and total dataset). Where $\mathrm{x}$-axis represents samples and $\mathrm{y}$-axis represents frequency.
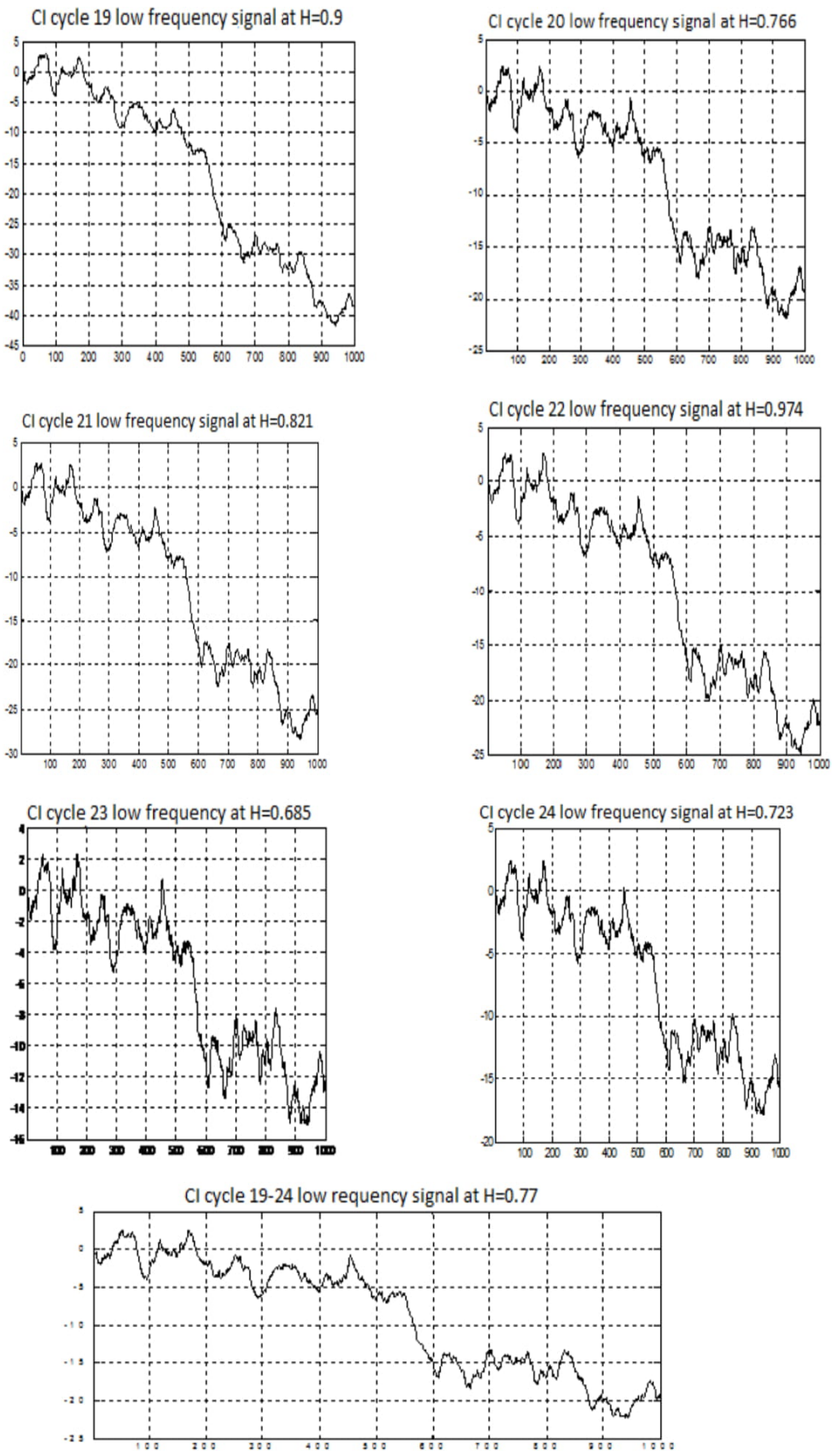
Fig.2 A Strong low-frequency signal is obtained with the help of Hurst value(H), in using ( $\mathrm{fBm})$, that shows the long term dynamics of NINO-3.4 region (cyclic and total dataset). Where $\mathrm{x}$-axis represents samples and $y$-axis represents frequency.
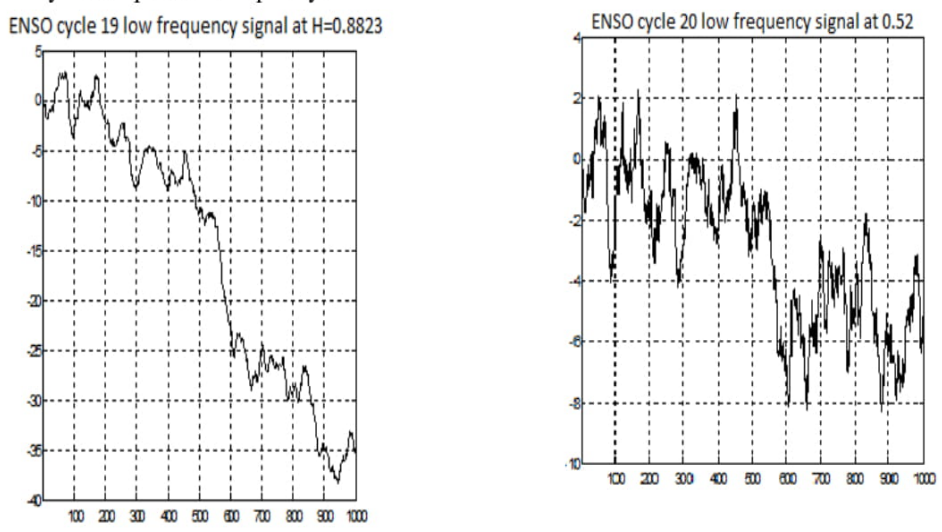

ENSO cycle 21 low frequency signal at $\mathrm{H}=0.586$
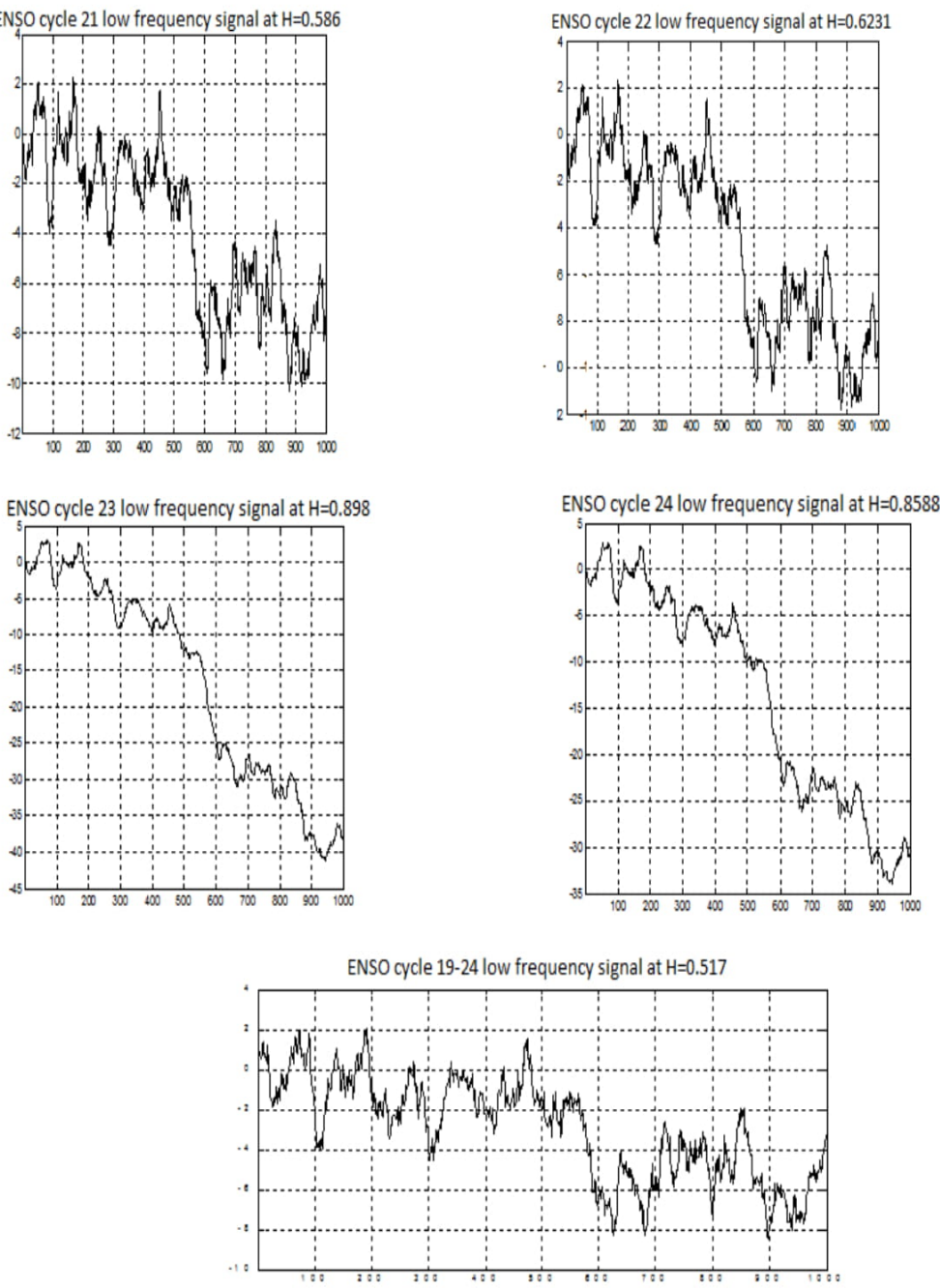
Fig. 3A strong low-frequency signal is obtained with the help of Hurst value(H), in using ( $(\mathrm{Bm})$, that shows the long term dynamics of QBO (cyclic and total dataset). Where X-represents samples and y-represents frequency.

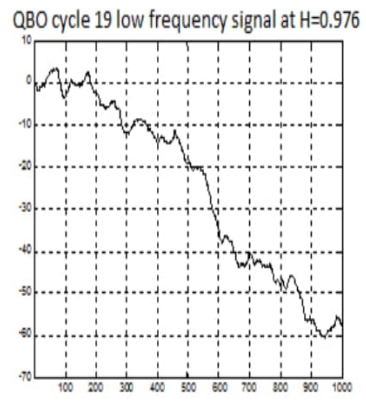

QBO cycle 21 low frequency signal at $\mathrm{H}=0.866$
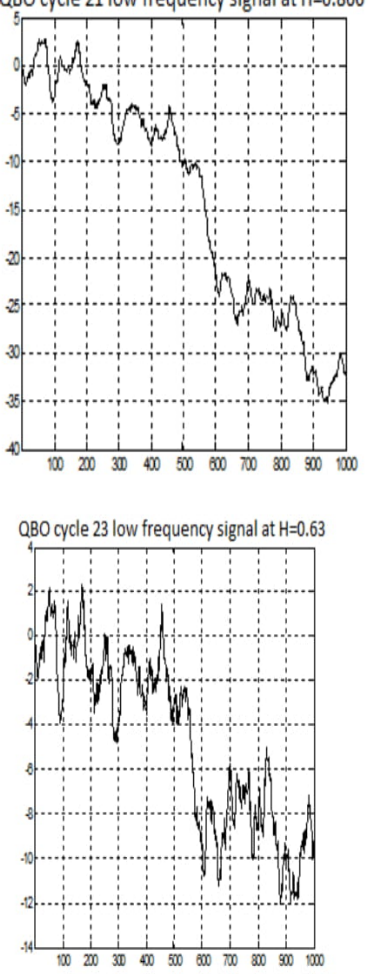

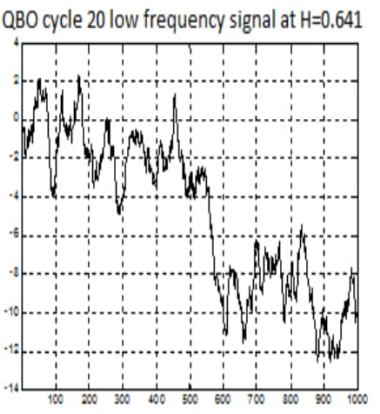

$\mathrm{QBO}$ cycle 22 low frequency signal at $\mathrm{H}=0.691$
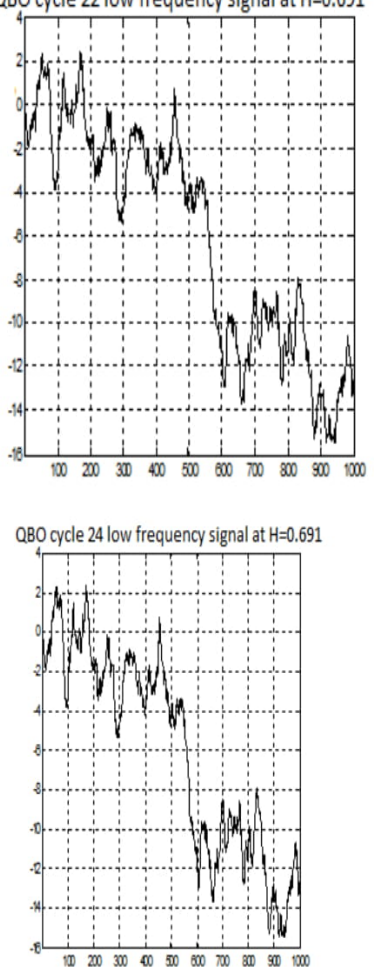

QBO cycle 19-24 low frequency signal at $\mathrm{H}=0.546$

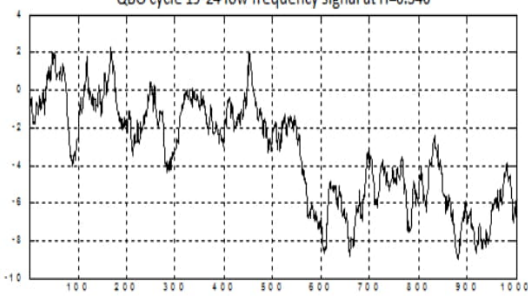




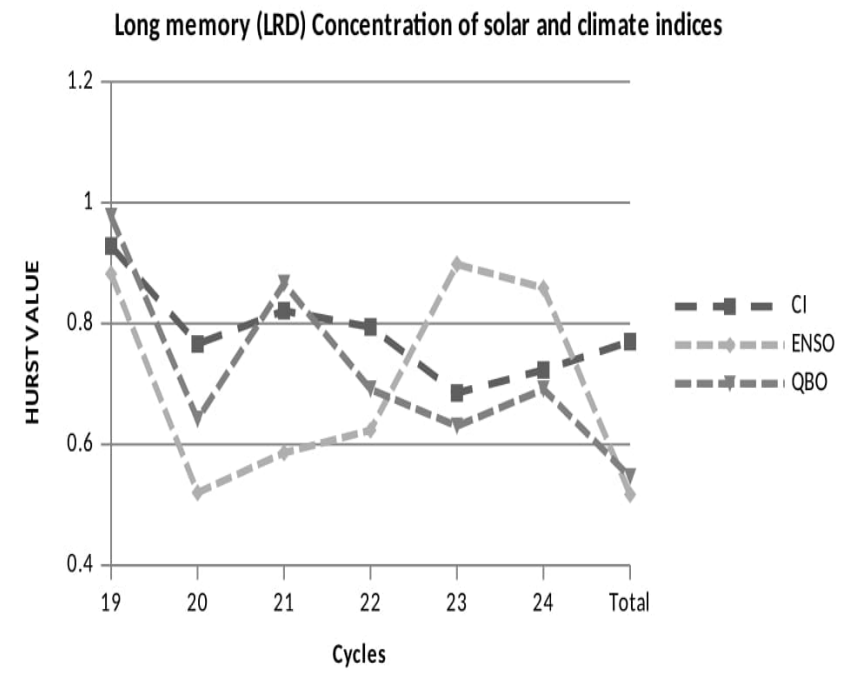

Fig. 4 Long memory dependency trend comparison of CI, ENSO and QBO (cycles and total dataset). 